\title{
Variabilité inter- et intra-individuelle de quelques caractéristiques anatomiques de l'écorce de l'épicéa commun (Picea abies (L.) Karsten) en Ardenne belge
}

\author{
P. Nihoul ${ }^{1}$, L. Nef ${ }^{1}$ et L. Waterkeyn²
}

1 Centre de lutte intégrée contre les insectes forestiers*, Unité des Eaux et Forêts, UCL, place Croix-du-Sud, 2, 1348 Louvain-La-Neuve, Belgique

2 Laboratoire de cytologie végétale, UCL, place Croix-du-Sud, 2, 1348 Louvain-La-Neuve, Belgique

(reçu le 9-3-1988; accepté le 8-7-1988)

Résumé - Cette étude aborde l'anatomie de l'écorce de l'épicéa commun en analysant la variabilité de ses structures qui pourrait rendre compte des variabilités dans l'intensité d'attaque par l'ips typographus. Ces structures ont été quantifiées sur une vingtaine d'épicéas de 2 peuplements localisés dans les Ardennes belges et, sur chaque arbre, à 3 hauteurs $(1,5,3$ et $6 \mathrm{~m}$ ) et à 2 orientations (nord et sud). Nous avons pris en considération les différents groupes tissulaires, le système résinifère, le sclérenchyme et les cellules à cristaux du phloème secondaire.

Les résultats sont les suivants :

- il y a une importante variabilité entre les arbres pour toutes les caractéristiques étudiées sauf en ce qui concerne l'importance du système résinifère. Pour cette dernière, au sein d'un des 2 peuplements étudiés, il n'apparaît aucune différence significative;

- les 3 hauteurs sont une source importante de variation pour les structures de l'écorce, excepté pour l'importance du système résinifère et la fréquence des cellules à cristaux;

- aucune caractéristique de l'écorce ne diffère significativement entre les 2 orientations, sauf pour la quantité de massifs sclérenchymateux dans un des 2 peuplements.

Dès lors l'écorce de l'épicéa présente une variabilité inter- et intra-individuelle qui pourrait être en relation avec des préférences de l'lps typographus pour certaines hauteurs et avec des différences de résistance entre arbres.

écorce - histologie - variabilité des structures - épicéa commun - Picea abies

Summary - Variability of bark anatomical characteristics between and within Norway spruce, Picea abies (L.) Karsten, in upper Belgium. This paper gives the results of a quantitative analy* Recherches subventionnées par I'IRSIA (Institut pour l'Encouragement de la Recherche Scientifique dans I'In-
dustrie et l'Agriculture). 
sis of the bark anatomical structures of the Norway spruce (Picea abies (L.) Karst.). The aim of the research was to find links between the different levels in the attack intensity by the bark beetle Ips typographus $L$. and the variability of the bark structure between and within trees.

The structures were quantified on 19 trees of 2 spruce stands located in the belgian Ardennes. Bark samples were taken from 3 heights (1.5-3-6 m) and 2 faces (north and south). On $40 \mu \mathrm{m}$ thick microscopic preparations, we quantified the different tissular groups, the resiniferous system, the secondary phloem sclerenchyma and the crystal cells.

All the analysed characteristics showed a marked variability between trees. Nevertheless, the importance of the resiniferous system was not significantly different among the spruces; this appeared only in one stand studied.

The 3 heights were a significant source of bark structure variability except for the transversal resiniferous system section and the crystal cell frequency.

No differences were found between the 2 orientations, except for the sclerenchyma volume. But in this case, the difference was lower than between the individuals or between the levels at which the samples were taken; furthermore, this difference existed only in one site.

In conclusion, the variability in bark structures between the trees appears to be important and further research on the insects' attacks is required.

\section{bark - histology - structure variability - Norway spruce - Picea abies}

\section{Introduction}

L'anatomie de l'écorce de l'épicéa commun (Picea abies (L.) Karst.), encore relativement mal connue, pourrait jouer un rôle majeur dans la résistance de l'arbre aux insectes corticoles tel l'Ips typographus L. (Coleoptera : Scolytidae).

Des degrés variables d'intensités d'attaque ont été observés dans un peuplement homogène entre des arbres sains rendus attractifs par un traitement aux phénomènes d'agrégation de l'insecte (Nef, en préparation). Des différences entre les écorces de ces arbres expliquent probablement cette variabilité.

Nous présentons ici les résultats de l'étude d'un premier volet d'une recherche sur le sujet, à savoir dans quelle mesure les structures anatomiques de l'écorce varient quantitativement et seront susceptibles, ultérieurement, d'être mises en relation avec la résistance aux insectes. Une autre étude a déjà abordé des paramètres chimiques (Janssens, 1987). Ultérieurement, nous rechercherons les corrélations existant entre ces divers paramètres et les attaques d'insectes.

Une description générale de l'écorce des conifères est donnée par Chang (1954), et une description du phloème secondaire, en particulier par Srivastava (1963). Quant à l'écorce de l'épicéa commun, elle est décrite par Eremin (1976, 1977) et Liese et Parameswaran (1971). L'ultrastructure de certains types cellulaires a aussi fait l'objet de quelques études (Wattendorff et Schmid, 1973; Parameswaran, 1975; Parameswaran et al., 1976; Vassiliev et Carde, 1976). Ces auteurs ne donnent cependant aucune indication sur l'importance quantitative des diverses structures, lacune que nous voudrions contribuer à combler.

La présente étude a pour but de quantifier des caractéristiques anatomiques et d'en analyser la variabilité entre les arbres d'un même peuplement et, sur un même arbre, entre 3 hauteurs et 2 orientations; ces caractéristiques sont : les différents groupes tissulaires, le système résinifère, le sclérenchyme et les cellules à cristaux du phloème secondaire. 


\section{Matériel et Méthodes}

L'étude a porté sur des épicéas choisis aléatoirement dans 2 peuplements équiennes de 70 et 50 ans environ, localisés respectivement à Vielsalm (Vs) et La Roche-en-Ardenne (LR). Sur 9 (Vs) ou 10 arbres (LR) de chaque peuplement, des échantillons d'écorce (21 $\mathrm{mm}$ de diamètre) ont été prélevés à 3 hauteurs : 1,5, 3 et $6 \mathrm{~m}$, et selon 2 orientations : nord et sud, en mai (Vs) et juillet (LR) 1986.

Les mesures sont toutes effectuées sur des coupes transversales de $40 \mathrm{~mm}$ d'épaisseur et d'une surface moyenne de $70 \mathrm{~mm}^{2}$. L'épaisseur radiale des différents tissus est une moyenne de 4 mesures, tous les $1,8 \mathrm{~mm}$, déterminée sur projection de la coupe. Nous avons aussi étudié sur coupe projetée le sclérenchyme dans la zone du liber s'étendant du cambium jusqu'à $\approx 3,20 \mathrm{~mm}$ de ce dernier, et les cellules à cristaux dans la zone entre = 0,87 et $1,75 \mathrm{~mm}$ à partir du cambium, c'est-àdire où l'lps typographus creuse ses galeries. La surface des massifs de sclérenchyme est assimilée à une ellipse dont on a mesuré les 2 axes principaux. Le système résinifère est dessiné avec une chambre claire au grossissement de $15 \mathrm{x}$, puis sa surface relative est mesurée à l'aide d'une table graphique.

Le traitement statistique des données( $\left.{ }^{\star}\right)$ a été réalisé par une analyse de la variance portant sur les 3 critères : arbre, hauteur et orientation.

\section{Résultats et discussion}

\section{Les principaux groupes tissulaires}

Les groupes tissulaires suivants ont été quantifiés (Figs. 1,2 et 3 ) : le ou les périderme(s); les tissus morts (rhytidome) : parenchyme cortical et/ou phloème secondaire; les tissus vivants de l'écorce interne.

Variabilité entre arbres (Tableau I). Les épaisseurs de ces groupes tissulaires dif-

(*) Nous sommes reconnaissants à Monsieur Pierre Dutilleul (Unité de biométrie, Faculté des Sciences Agronomiques, Université Catholique de Louvain) qui nous a aidé efficacement.

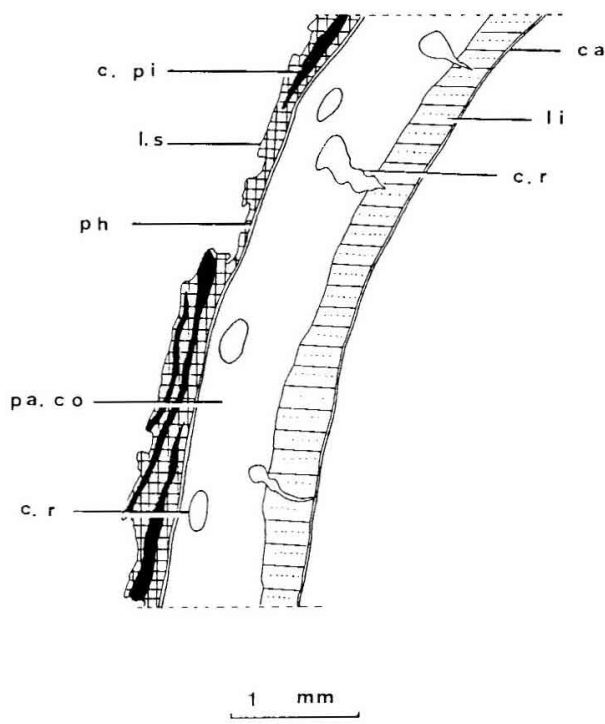

Fig. 1. Ecorce d'une pousse de 6 ans. Coupe transversale. Vue d'ensemble. ca : cambium; $c$. pi : cellules pierreuses; c.r. : canal résinifère; I : liège; I.s. : liège spongieux; li : liber; pa. co : parenchyme cortical; ph : phellogène et phelloderme; sc : sclérenchyme.

fèrent d'un arbre à l'autre, significativement $(P<0,05)$ ou très significativement $(P<0,01)$.

Ces différences histologiques de l'écorce entre des épicéas de même âge résultent de la formation, dans les tissus préexistants, de nouveaux phellogènes :

a. Des vieilles écorces, à $1,5 \mathrm{~m}$ de hauteur sur les arbres de 50 et 70 ans, peuvent encore avoir un périderme unique formé par ce premier phellogène. Ce dernier, déjà présent dans l'écorce de la pousse d'une année (nous n'avons observé aucun nouvel histogène dans les rameaux de 1 à 6 ans), reste en activité pendant de nombreuses années.

b. De nouveaux phellogènes peuvent se succéder, apparemment à un rythme non défini et relativement lent. II y a généralement un ou 2 péridermes, rarement 3. Cette néoformation d'un nouveau méristème ne se réalise pas de façon continue 


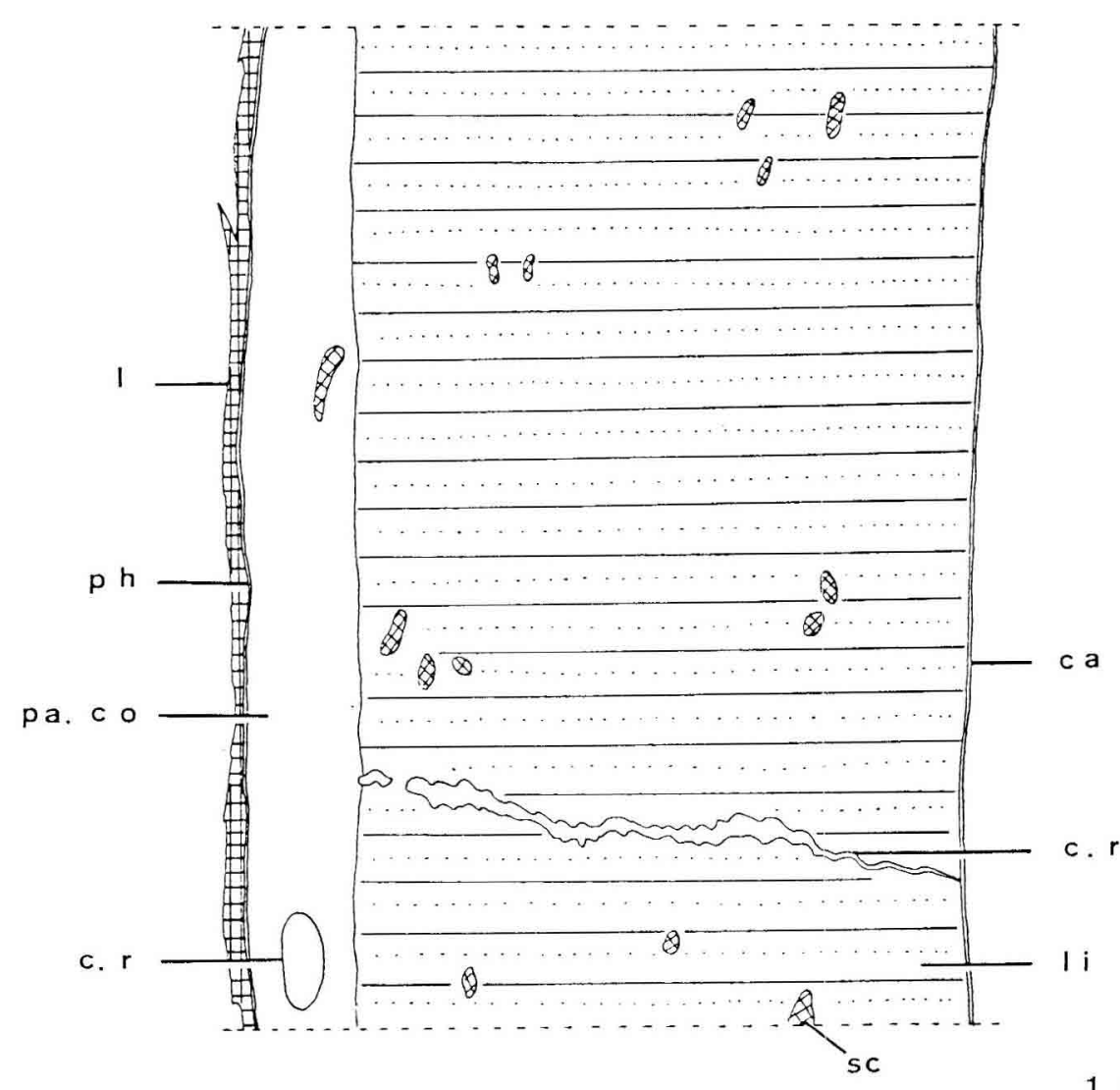

Fig. 2. Ecorce d'un arbre de 70 ans. Coupe transversale. Vue d'ensemble (prélèvement à $6 \mathrm{~m})$. Légende : voir Fig. 1.

sur tout le périmètre du tronc; seul un faible volume de tissus corticaux est isolé par le nouveau périderme et forme une sorte de lentille ou d'écaille. De la sorte, peuvent se jouxter des rhytidomes d'épaisseurs différentes, résultant d'un nombre différent de péridermes.

c. Le nouveau phellogène ne prend pas naissance directement sous le précédent pour former une succession continue de péridermes, mais il se forme plus ou moins profondément, isclant une épaisseur var riable de tissus comprise entre $40 \mu \mathrm{m}$ et $4000 \mu \mathrm{m}$.

En conclusion, pour des épicéas d'un même peuplement, la formation de nouveaux péridermes et, conséquemment, d'un rhytidome plus épais, est propre à chaque individu. Certains sujets présentent un rhytidome finement écailleux, dû à la desquamation des lentilles de cellules pierreuses appartenant à l'unique périder- 


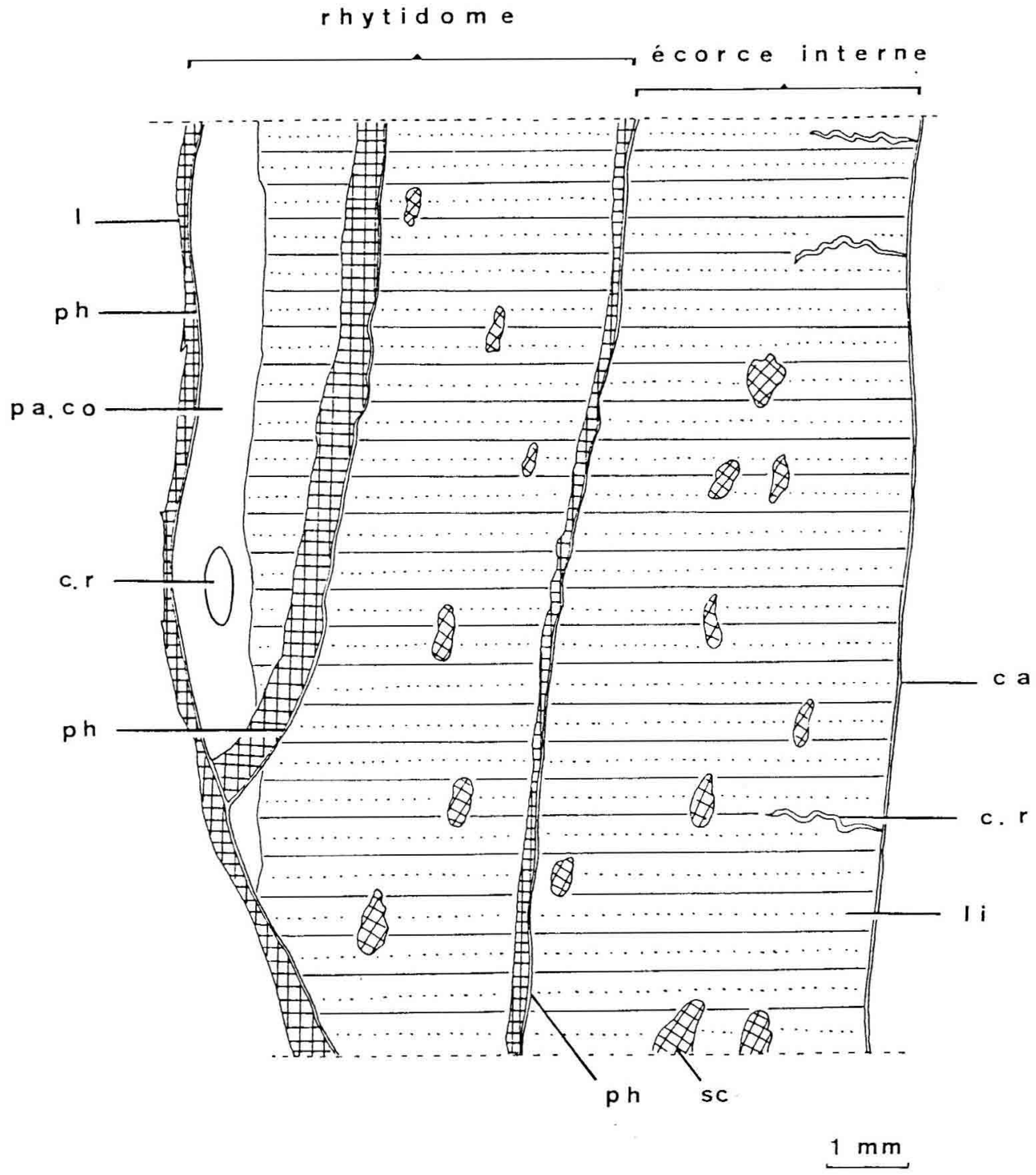

Fig. 3. Ecorce d'un arbre de 70 ans. Coupe transversale. Vue d'ensemble (prélèvement à 1,5 m). Légende : voir Fig. 1.

me externe: tandis que d'autres ont un macrorelief extérieur assez tourmenté, suite à la formation de péridermes isolant des écailles de tissus morts. Les premiers ont un pourcentage élevé de tissus vivants (jusqu'à $97 \%$ en moyenne pour les 3 hauteurs), alors que pour les autres, celui-ci ne dépasse pas $62 \%$. 
Variabilité due à la hauteur (Tableau II). Des différences significatives $(P<0,05$ ou $<0,01$ ) apparaissent entre les 3 hauteurs pour ces mêmes caractéristiques. L'épaisseur du rhytidome diminue avec la hauteur, c'est-à-dire avec l'âge, alors que le phénomène opposé se présente pour les tissus vivants de l'écorce interne. Quant au pourcentage de tissus vivants à $6 \mathrm{~m}$, il est en moyenne de $95,8 \%$ (Vs) ou $89,5 \%$ (LR), mais n'est plus que de $76,7 \%$ (Vs) ou $76,4 \%$ (LR) à $1,5 \mathrm{~m}$. A cette hauteur, le pourcentage d'écorce interne peut être très faible (25 à 30\%).

Variabilité due à l'orientation. Aucune différence significative n'est apparue entre les orientations nord et sud.
Le système résinifère (Figs. 4 et 6 )

\section{Variations quantitatives}

Variations entre arbres. Aucune différence significative n'a pu être mise en évidence entre les arbres du peuplement de Vielsalm, contrairement à celui de La Roche (où $P<0,01$ ). Pour ce dernier, le système résinifère actif représente en moyenne $11,8 \mu \mathrm{m}^{2} / 1000 \mu \mathrm{m}^{2}$ contre $6,5 \mu \mathrm{m}^{2}$ / $1000 \mu \mathrm{m}^{2}$ à Vielsalm : les 2 peuplements paraissent différents sur la base de cette caractéristique. II pourrait en être de même pour leur susceptibilité aux scolytes corticoles. Notre dispositif ne permet pas de montrer dans quelle mesure cette variabilité est imputable aux conditions

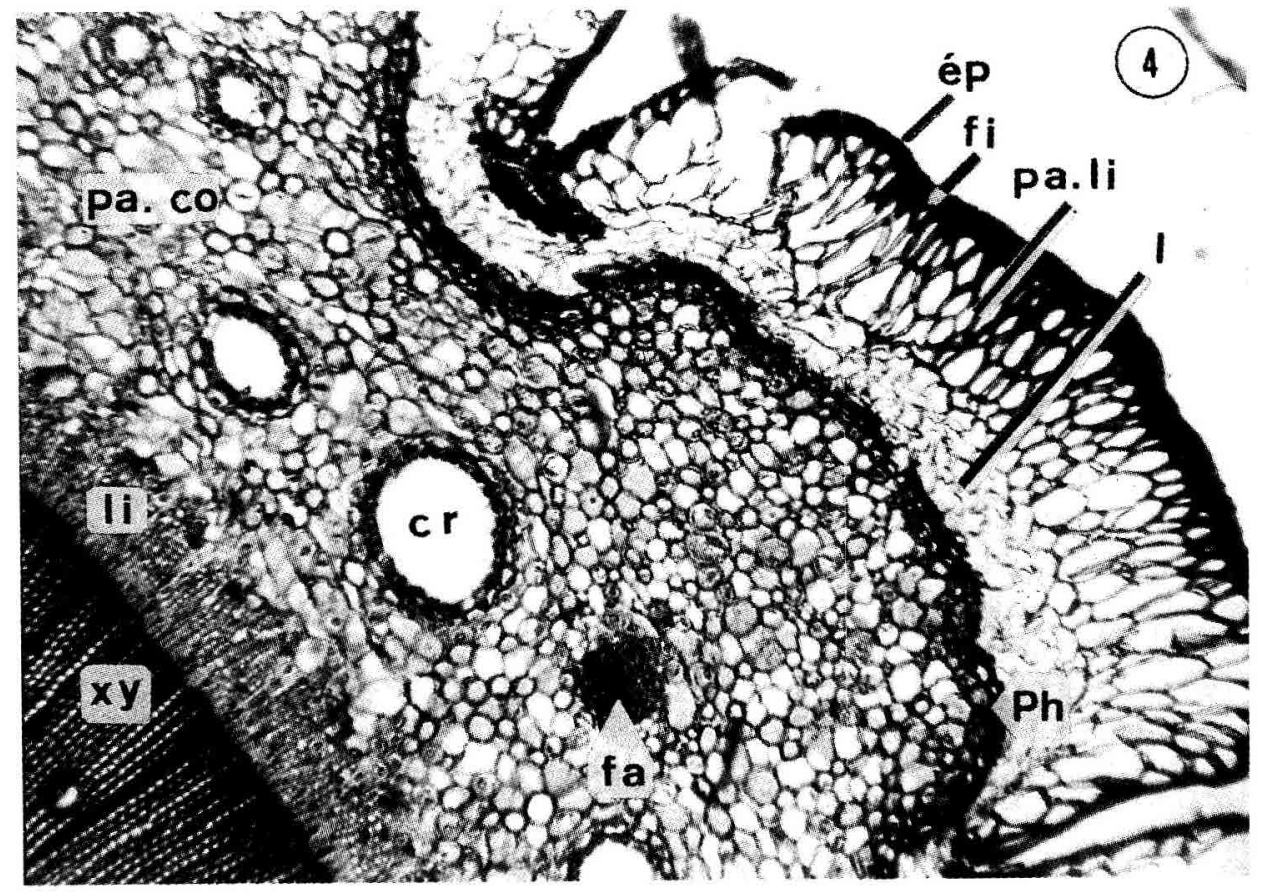

Fig. 4. Jeune pousse de un an; secteur en coupe transversale. 70 x. c.r. : canal résinifère; ép : épiderme; fa faisceau conducteur; fi : fibres; I : liège; li : liber; pa. co : parenchyme cortical; pa. li : parenchyme lignifié; ph : phellogène et phelloderme; $x y$ : xylème secondaire. 


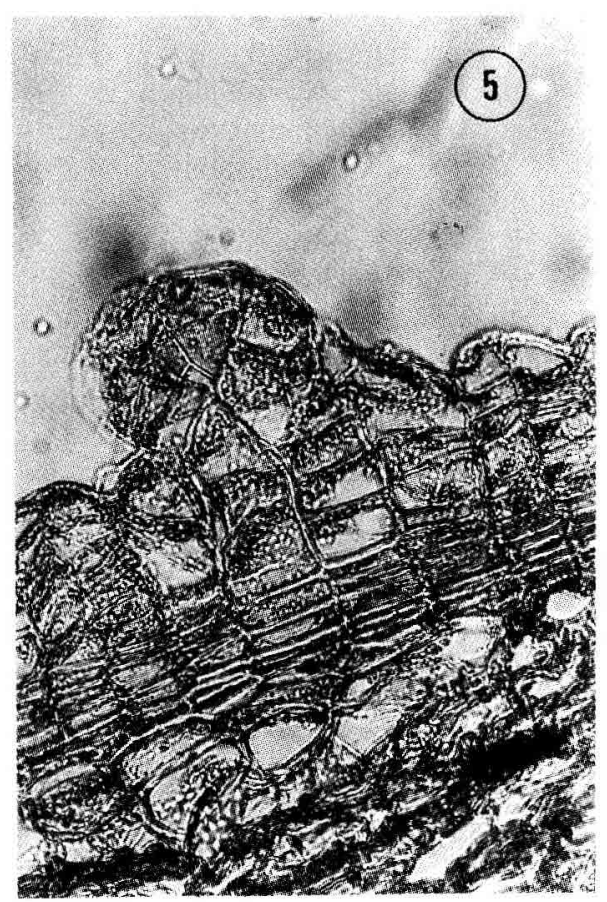

Fig. 5. Canal résinifère vertical : cellules bordant la cavité. Coupe transversale. $170 \mathrm{x}$.

environnementales ou aux caractéristiques génétiques.

Variabilité au sein d'un arbre. L'importance du système résinifère ne varie pas significativement, ni d'après les hauteurs de 1,5 , 3 et $6 \mathrm{~m}$, ni d'après l'orientation nord ou sud. Cependant, si des différences de volume de canaux dans l'écorce ne sont pas notées, il n'est pas impossible que la quantité de résine sécrétée puisse varier, comme l'ont montré Christiansen et Horntvedt (1983).

\section{Le rôle défensif}

La littérature attribue au système résinifère de l'écorce un rôle important dans la défense du conifère vis-à-vis des insectes corticoles; le système préformé de résistance, c'est-à-dire préexistant à l'attaque, reposerait uniquement sur ces canaux (Berryman, 1972). Cet effet défensif serait dû à l'écoulement de résine qui peut avoir un effet répulsif mécanique, et à une action dissuasive, voire toxique, due à ses composants chimiques. En particulier, le système de résistance de l'épicéa contre l'attaque de l'/ps typographus et des champignons du genre Ceratocystis que ce dernier véhicule dépendrait, en partie du moins, de la résine s'écoulant des canaux résinifères endommagés (Christiansen et Horntvedt, 1983).

\section{Description}

L'épicéa possède des canaux résinifères horizontaux et verticaux (Chang, 1954). Concernant ces derniers, nous avons pu

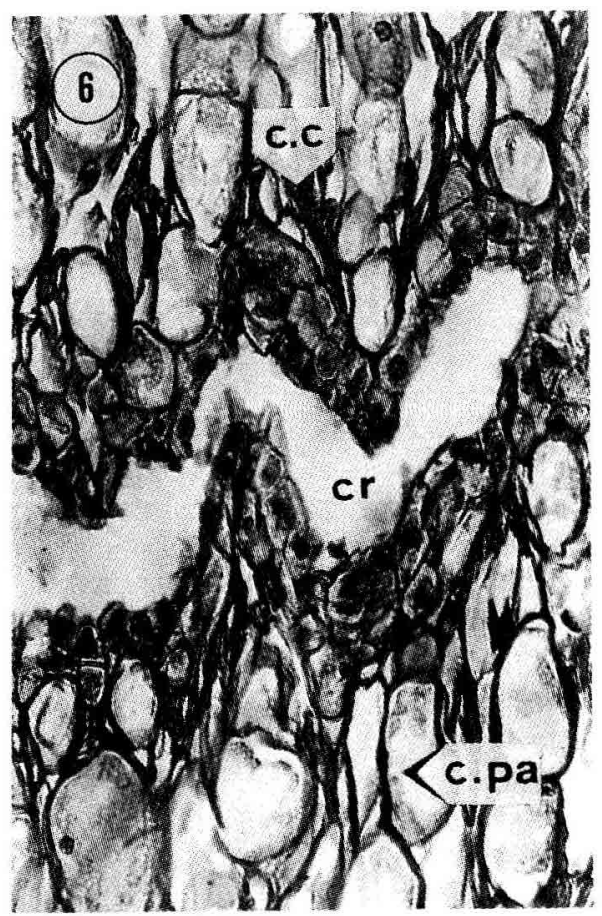

Fig. 6. Canal résinifère sinuant plus ou moins radialement dans le phloème âgé. Coupe transversale. $170 \mathrm{x}$. c.c : cellule criblée; c. pa : cellule de parenchyme; $\mathrm{cr}$ : canal résinifère. 
ajouter des informations nouvelles à celles de la littérature (Eremin, 1975).

Les canaux verticaux déjà formés dans le parenchyme vertical de la jeune pousse d'une année (Fig. 4) demeurent dans l'écorce âgée tant que ce tissu ne fait pas partie du rhytidome, puis subissent des modifications au cours de la croissance de l'écorce :

1. ils perdent leur rectitude juvénile, de sorte qu'ils peuvent sillonner obliquement l'écorce primaire. Ainsi, des coupes longitudinales radiales peuvent les faire apparaître sous forme de cavités elliptiques. $\mathrm{Ce}$ fait est dû aussi à des dilatations locales du canal qui font ainsi penser à des poches. Découlant de ce fait, les canaux ont une section plus importante que dans les jeunes pousses. Ils peuvent atteindre $2 \mathrm{~mm}$ dans la direction tangentielle, contre $0,7 \mathrm{~mm}$ radialement;

2. le nombre d'assises épithéliales sécrétrices, formant l'épaisseur de la paroi du canal, s'est fortement accru : de 1 à 2 dans de jeunes pousses, il passe à plus d'une dizaine pour des canaux plus âgés. Leur disposition aussi est particulière (Fig. 5) : elles forment des files bien alignées, comme si elles dérivaient d'une assise méristématique. Toutefois, cet alignement peut s'estomper plus ou moins pour les cellules les plus proches de la lumière du canal. Localement, par division cellulaire, des proliférations forment un léger relief interne.

En outre, en section transversale, les cellules les plus internes diffèrent des plus internes. Leur forme rectangulaire est plus marquée et leur lumen est moins important. Elles présentent dès lors un aspect nettement plus aplati. Ces caractéristiques sont parfois moins marquées.

Enfin, le nombre de canaux verticaux dans l'écorce âgée est nettement supérieur à celui d'une jeune pousse. De nouveaux canaux doivent donc se former pen- dant la croissance sur la circonférence. Mais il y a aussi des canaux qui deviennent inactifs; leur lumen se remplit de cellules, suite à la formation de sortes de tylles, et possèdent des cellules épithéliales ressemblant à un périderme.

Le sclérenchyme et les cellules à cristaux du liber

L'épaisseur moyenne des parois des cellules sclérenchymateuses est de l'ordre de $25 \mu \mathrm{m}$; elles apparaissent dès la cinquième année et forment des amas qui peuvent occuper jusqu'à $15 \%$ de la surface du liber. Le nombre de cellules à cristaux qui se forment surtout dans l'ancien phloème fluctue entre 2 et 30 par $\mathrm{mm}^{2}$ de section transversale. Ainsi une hypothèse serait que ces structures constituent une défense mécanique contre les insectes corticoles.

\section{Le sclérenchyme}

Variabilité entre arbres. II y a des différences importantes $(P<0,01)$ entre les arbres de chaque peuplement (Tableau I) : dans un peuplement équienne, les épicéas n'ont donc pas un même volume de sclérites dans leur liber.

Variabilité due à la hauteur. Les volumes d'amas sclérenchymateux diffèrent très significativement $(P<0,01)$ d'après la hauteur. Le volume le plus important se trouve à la base du tronc et diminue avec la hauteur, cette diminution étant plus forte entre 1,5 et $3 \mathrm{~m}$ qu'entre 3 et $6 \mathrm{~m}$ (Tableau II) : la différenciation n'aurait donc lieu qu'à partir d'un certain âge.

Variabilité due à l'orientation. Une différence entre le nord et le sud n'est décelable que pour le peuplement de La Roche $(P<0,05)$ où la moyenne au nord est de $75 \mu \mathrm{m}^{2 / 1} 000 \mu \mathrm{m}^{2}$ (écart type $\sigma=15,1$ $\mu \mathrm{m}^{2} / 1000 \mu \mathrm{m}^{2}$ ) contre $54,2 \mu \mathrm{m}^{2 / 1} 000 \mu \mathrm{m}^{2}$ 
Tableau I. Variabilité de caractéristiques étudiées en fonction des individus.

\begin{tabular}{|c|c|c|c|c|c|c|c|c|}
\hline & \multicolumn{2}{|c|}{ \% par. lib. v. } & \multicolumn{2}{|c|}{ Cellules à cristaux } & \multicolumn{2}{|c|}{ Sclérenchyme } & \multicolumn{2}{|c|}{ Syst. résin. act } \\
\hline & $m$ & $C V$ & $m$ & $\mathrm{CV}$ & $m$ & $C V$ & $m$ & $c V$ \\
\hline \multicolumn{9}{|c|}{ Dispositif de Vielsalm } \\
\hline 1 & 73,5 & 18,0 & 18,9 & 20,2 & 73,5 & 62,3 & 4,4 & 115,1 \\
\hline 2 & 94,2 & 5,8 & 15,9 & 21,6 & 53,8 & 109,4 & 6,5 & 50,5 \\
\hline 3 & 91,8 & 13,6 & 10,2 & 32,2 & 54,3 & 71,2 & 5,0 & 84,9 \\
\hline 4 & 87,6 & 15,5 & 47,9 & 37,1 & 26,5 & 160,7 & 10,4 & 41,6 \\
\hline 5 & 92,0 & 10,2 & 35,5 & 27,1 & 10,1 & 159,9 & 12,9 & 69,6 \\
\hline 6 & 73,4 & 28,5 & 8,8 & 54,4 & 66,8 & 91,7 & 6,4 & 86,6 \\
\hline 7 & 84,7 & 24,6 & 24,4 & 12,8 & 1,3 & 244,9 & 5,3 & 51,6 \\
\hline 8 & 58,8 & 51,5 & 7,3 & 38,5 & 25,9 & 148,6 & 3,5 & 86,7 \\
\hline 9 & 94,6 & 5,9 & 6,4 & 32,4 & 44,4 & 53,6 & 4,4 & 146,3 \\
\hline$P$ & \multicolumn{2}{|c|}{ ** } & \multicolumn{2}{|c|}{ ** } & \multicolumn{2}{|c|}{$\star \star \infty \quad 0,0$} & \multicolumn{2}{|c|}{ NS } \\
\hline \multicolumn{9}{|c|}{ Dispositif de La Roche } \\
\hline 1 & 62,3 & 29,7 & 13,9 & 55,6 & 42,3 & 117,4 & 3,5 & 98,6 \\
\hline 2 & 88,0 & 14,4 & 4,0 & 32,6 & 145,3 & 54,2 & 15,0 & 66,3 \\
\hline 3 & 89,0 & 14,7 & 15,9 & 59,0 & 120,8 & 37,8 & 12,6 & 68,4 \\
\hline 4 & 80,8 & 22,3 & 5,7 & 52,4 & 77,5 & 98,7 & 6,5 & 99,3 \\
\hline 5 & 90,0 & 14,8 & 10,6 & 42,3 & 40,0 & 57,5 & 6,7 & 52,6 \\
\hline 6 & 97,3 & 0,5 & 13,4 & 24,6 & 134,2 & 52,2 & 19,3 & 55,9 \\
\hline 7 & 84,8 & 20,9 & 20,2 & 19,4 & 8,1 & 111,1 & 19,3 & 50,8 \\
\hline 8 & 83,5 & 23,3 & 5,7 & 33,9 & 12,5 & 170,8 & 10,4 & 53,8 \\
\hline 9 & 89,0 & 16,8 & 6,2 & 46,3 & 31,6 & 110,9 & 20,9 & 47,0 \\
\hline 10 & \multirow{2}{*}{\multicolumn{2}{|c|}{93,3 ** }} & 11,7 & 32,4 & 33,6 & 42,2 & 3,8 & 80,8 \\
\hline$P$ & & & \multicolumn{2}{|r|}{ U, } & \multicolumn{2}{|c|}{ ** } & \multicolumn{2}{|c|}{$\star \star$} \\
\hline
\end{tabular}

\% par. lib.v. : pourcentage de parenchyme et/ou liber vivant. Cellules à cristaux : nombre de cellules à cristaux par $\mathrm{mm}^{2}$ de section transversale. Sciérenchyme : surface du sclérenchyme en $\mu \mathrm{m}^{2} / 1000 \mu \mathrm{m}^{2}$ de section transversale. Syst. résin. act. : surface du système résinifère actif en $\mu \mathrm{m}^{2}: 100 \mu \mathrm{m}^{2}$ de section transversale. Moyenne $(m)$ et coefficient de variation (CV) pour les arbres. Niveaux de signification des différences entre les arbres : $\mathrm{NS}=$ non significatif; ${ }^{*}=P<0,01$.

$\left(\sigma=58,3 \mu \mathrm{m}^{2 / 1} 000 \mu \mathrm{m}^{2}\right)$ au sud. Cet effet orientation est sensiblement moins important que les effets «individu» et «hauteur».

\section{Les cellules à cristaux}

Variabilité entre les arbres. II existe de fortes différences $(P<0,01)$ entre les arbres de chaque peuplement (Tableau I); la moyenne pour le peuplement de Vielsalm est de 19,5 cellules à cristaux par $\mathrm{mm}^{2}\left(\sigma=15,1 / \mathrm{mm}^{2}\right)$ contre $10,7 / \mathrm{mm}^{2}(\sigma$ $\left.=6,7 / \mathrm{mm}^{2}\right)$ à La Roche.

Variabilité au sein d'un arbre. Aucune différence significative ne fut trouvée ni entre les 3 hauteurs, ni entre les 2 orientations.

\section{Conclusion}

Entre les épicéas d'un même peuplement équienne et sain, toutes les caractéristiques étudiées présentent une variabilité importante, la seule exception étant le système résinifère dont l'importance ne paraît pas différer entre les arbres du peuplement de Vielsalm.

Au sein d'un arbre, il n'y a pas de différence entre les orientations nord et sud. Mais des différences apparaissent entre les 3 hauteurs, excepté pour les cellules à cristaux du liber et pour le système résinifère, tandis qu'elles sont prononcées pour l'épaisseur des différents groupes tissu- 
Tableau II. Variabilité de caractéristiques étudiées en fonction des individus.

\begin{tabular}{|c|c|c|c|c|c|c|c|}
\hline & \multicolumn{2}{|c|}{$\begin{array}{c}\text { Hauteur en } m \\
\qquad 1,5\end{array}$} & \multicolumn{2}{|c|}{3} & \multicolumn{2}{|c|}{6} & \multirow[t]{2}{*}{$P$} \\
\hline & $m$ & $C V$ & $m$ & $\mathrm{CV}$ & $m$ & $C V$ & \\
\hline \multicolumn{8}{|c|}{ Dispositif de Vielsalm } \\
\hline Périderme & 468,4 & 53,9 & 388,1 & 31,9 & 251,7 & 47,3 & ** \\
\hline Par. lib. m. & 1358,5 & 144,2 & 1259,4 & 88,4 & 39,3 & 399,2 & * \\
\hline Rhytidome & 1826,9 & 118,8 & 1647,5 & 73,5 & 291,0 & 79,3 & * \\
\hline Par. lib.v. & 5715,3 & 33,1 & 5755,5 & 21,1 & 6770,5 & 9,9 & * \\
\hline \% par. lib. v. & 76,7 & 33,0 & 77,9 & 20,3 & 95,8 & 3,4 & ** \\
\hline Sclérenchyme & 75,3 & 66,9 & 25,1 & 103,6 & 18,5 & 162,7 & ** \\
\hline C. à cristaux & 18,3 & 86,9 & 22,3 & 79,8 & 17,8 & 64,6 & NS \\
\hline Syst. rés. act. & 6,2 & 75,4 & 5,5 & 72,2 & 7,8 & 96,9 & NS \\
\hline \multicolumn{8}{|c|}{ Dispositif de La Roche } \\
\hline Périderme & 378,8 & 36,9 & 240,4 & 42,3 & 220,2 & 43,4 & ** \\
\hline Par. lib. m. & 1266,2 & 98,9 & 332,4 & 199,7 & 478,8 & 188,7 & ** \\
\hline Rhytidome & 1643,0 & 82,4 & 572,9 & 128,5 & 699,1 & 140,4 & ** \\
\hline Par. lib. v. & 5233,8 & 28,3 & 6264,6 & 17,6 & 6060,9 & 21,4 & ** \\
\hline \% par. lib. v. & 76,4 & 24,5 & 91,5 & 12,1 & 89,5 & 16,5 & ** \\
\hline Sclérenchyme & 103,7 & 79,6 & 51,6 & 99,4 & 38,4 & 111,4 & ** \\
\hline C. à cristaux & 9,8 & 70,4 & 11,9 & 64,7 & 10,4 & 51,9 & NS \\
\hline Syst. rés. act. & 11,8 & 76,1 & 10,8 & 84,3 & 12,8 & 83,3 & NS \\
\hline
\end{tabular}

Périderme : épaisseur en mm du périderme. Par. lib. m. : épaisseur en $m$ du parenchyme etvou liber mort. Par. lib. v. : épaisseur en $m$ du parenchyme et/ou liber vivant. Rhytidome : épaisseur en m du rhytidome. Sclérenchyme : surface du sclérenchyme en $\mu \mathrm{m}^{2} / 1000 \mu \mathrm{m}^{2}$ de section transversale. C. à cristaux : nombre de cellules à cristaux par $\mathrm{mm}^{2}$ de section transversale. Syst. rés. act. : surface du système résinifère actif en $\mu \mathrm{m}^{2} / 1000 \mu \mathrm{m}^{2}$ de section transversale. Moyenne $(m)$ et coefficient de variation (CV). Niveaux de signification des differences entre les hauteurs $:$ NS $=$ non significatif; ${ }^{*}=P<0,05 ;{ }^{* *}=P<0,01$.

laires et la fréquence du sclérenchyme dans le phloème secondaire.

Dès lors, tous les épicéas ne présentent pas un même substrat pour les insectes corticoles. Des niveaux de sensibilité ou de résistance différents d'un arbre à l'autre pourraient s'expliquer, au moins partiellement, par cette variabilité anatomique inter-individuelle de l'écorce, tout comme les différences intra-individuelles pourraient expliquer les préférences des scolytes envers certaines hauteurs comme sites de ponte.

\section{Références}

Berryman A. (1972) Resistance of conifers to bark beetle-fungus associations. Bioscience 22 , 598-602
Chang HY.P. (1954) Bark structure of north american conifers. US Dep. Agric. Tech. Bull. 1095, 1-86

Christiansen E. \& Horntvedt R. (1983) Combined $/ p s / C e r a t o c y s t i s$ attack on Norway spruce and defensive mechanisms of the trees. $Z$. Angew. Entomol. 96, 110-118

Eremin V.M. (1975) Anatomie du système résinifère de l'écorce de quelques sapins, épicéas, mélèzes et pins. Biol. Nauki 18, 52-58 (en russe)

Eremin V.M. (1976) Anatomie de l'écorce d'espèces d'épicéas en URSS. Bot. Z. 20, 5-10 (en russe)

Eremin V.M. (1977) Caractéristiques de la structure anatornique de l'écorce de différentes conformations de Picea exelsa. Izv. Vyssh. Uchebn. Zaved. Les. Zh. 20, 5-10 (en russe)

Janssens R. (1987) Lutte intégrée contre Ips typographus. Facteur de résistance chez l'épicéa. UCL, Rapport Région Wallonne, 19861987 
Liese W. \& Parameswaran N. (1971) Über die Rindenanatomie starkborkiger Fichten. Forstwiss. Centralbl. 90, 370-375

Nef L. (1988) Quantification de l'intensité des attaques d'lps typographus L. sur Picea abies Karst (en préparation)

Parameswaran N. (1975) Zur Wandstruktur von Sklereiden in einigen Baumrinden. Protoplasma 85, 305-314

Parameswaran N., Kruse J. \& Liese W. (1976) Aufbau und Feinstruktur von Periderm und Len- ticellen der Fichtenrinde. Z. Pflanzenphysiol. $77,212-221$

Srivastava L.M. (1963) Secondary phloem in the Pinaceae. Univ. Calif. Publ. Bot. 36, 1-69

Vassiliev A.E. \& Carde J.P. (1976) Effet du gemmage sur l'ultrastructure des cellules sécrétrices des canaux de l'écorce des tiges de Pinus sylvestris L. et Picea abies (L.) Karst. Protoplasma 89, 41-48

Wattendorff J. \& Schmid H. (1973) Prüfung auf perjodatreaktive Feinstrukturen in den suberinisierten Kristallzellwänden der Rinde von Larix und Picea. Z. Pflanzenphysiol. 68, 422-431 